\title{
Determinants of Cutaneous Injection-Related Infections Among Injection Drug Users at an Emergency Department
}

\author{
Elisa Lloyd-Smith ${ }^{1,2}$, Mark Tyndall ${ }^{1,2,3}$, Ruth Zhang ${ }^{1}$, Eric Grafstein ${ }^{4}$, Sam Sheps ${ }^{2}$, Evan Wood ${ }^{1,2,3}$, \\ Julio Montaner ${ }^{1,3}$ and Thomas Kerr ${ }^{*}, 1,3$
}

\author{
${ }^{I}$ British Columbia Centre for Excellence in HIV/AIDS, Providence Health Care, 608-1081 Burrard Street, Vancouver, \\ V6Z 1Y6, Canada \\ ${ }^{2}$ School of Population and Public Health, University of British Columbia, 2206 East Mall, Vancouver, V6T 1Z3, Canada \\ ${ }^{3}$ Department of Medicine, University of British Columbia, 10203-2275 Laurel Street, Vancouver, V5Z 1M9, Canada \\ ${ }^{4}$ Department of Emergency Medicine, University of British Columbia, 855 West $12^{\text {th }}$ Ave, Vancouver, V5Z 1M9, Canada
}

\begin{abstract}
Introduction: Cutaneous injection-related infections (CIRI) are a primary reason injection drug users (IDU) access the emergency department (ED).

Methodology: Using Cox proportional hazard regression, we examined predictors of ED use for CIRI, stratified by sex, among 1083 supervised injection facility (SIF) users.

Results: Over a four-year period, 289 (27\%) visited the ED for CIRI, yielding an incidence density for females of 23.8 (95\% confidence interval (CI): 19.3 - 29.0) and males of 19.2 per 100 person-years (95\% CI: 16.7 - 22.1). Factors associated with ED use for CIRI among females included residing in the Downtown Eastside (DTES) (adjusted hazard ratio $[\mathrm{AHR}]=2.06[1.13-3.78]$ ) and being referred to hospital by SIF nurses (AHR = $4.48[2.76-7.30])$. Among males, requiring assistance with injection $(\mathrm{AHR}=1.38[1.01-1.90])$, being HIV-positive (AHR $=1.85[1.34-2.55])$, and being referred to hospital by SIF nurses $(\mathrm{AHR}=2.97$ [1.93 - 4.57]) were associated with an increased likelihood of an ED visit for CIRI.
\end{abstract}

Conclusion: These results suggest SIF nurses have facilitated referral of hospital treatment for CIRI, highlighting the need for continued development of efficient and collaborative efforts to reduce the burden of CIRI.

Keywords: Abscesses, cellulitis, emergency department, epidemiology, gender, health services, injection drug use.

\section{INTRODUCTION}

Cutaneous injection-related infections (CIRI), abscesses and cellulitis are a primary reason why people who inject drugs (IDU) access the Emergency Department (ED) [1-4]. Dramatic increases of hospital admissions for CIRI have been reported in several settings $[5,6]$. For example, in the United Kingdom, from 1997-1998 to 2003-2004, the number of abscess cases increased $566 \%$, while the number of cellutitis cases increased 469\% [5]. A recent study by Hope et al. highlighted that health care costs associated with these infections is high, conservatively ranging from $£ 15.5$ to $£ 30.0$ million annually [7]. Collectively, there is increasing awareness that improvement in treatment paradigms for CIRI is warranted [7].

In order for relevant and appropriate improvements in treatment of CIRI at the ED to occur, an understanding of the population using the ED for CIRI care is required. The present study was conducted to examine predictors of ED use for CIRI among IDU using a supervised injection facility

Address correspondence to this author at the Urban Health Research Initiative, BC Centre for Excellence in HIV/AIDS, 608-1081 Burrard Street, Vancouver, B.C. V6Z 1Y6, Canada; Tel: (604) 806-9116; Fax: (604) 8069044; E-mail: uhri@cfenet.ubc.ca
(SIF) in Vancouver, Canada. Since one of the objectives of the evaluation of the SIF was to assess the impact of the SIF on uptake of various treatment services [8-11], we also sought to assess whether the SIF is facilitating treatment for CIRI. Further, it has been previously shown that female IDU are more likely to develop CIRI $[2,12]$ and to be hospitalized $[13,14]$ and so we also sought to compare the incidence and risk factors for ED use for CIRI between female and male IDU.

\section{MATERIALS AND METHODS}

\section{Study Setting}

The SIF provides a service whereby IDU can inject preobtained illicit drugs under the supervision of medical staff $[15,16]$. Within SIF, individuals are provided with sterile injecting equipment, emergency intervention in the event of an overdose, plus routine primary medical care as well as addiction treatment, either on site or through referral [17]. North America's first government-sanctioned SIF opened in Vancouver, Canada, in September 2003. The SIF is located in the Downtown Eastside (DTES), which is home to many of Vancouver's IDU and is one of the poorest urban postal zones in Canada. The SIF has been designed to minimize barriers to medical care utilization among attendees, including onsite nursing care and referrals to other health 
and detox services [17]. St. Paul's Hospital is a public, acute care hospital with approximately 550 beds. It is the hospital in Vancouver that is in closest proximity to the DTES and is characterized by serving this high-risk population.

\section{Study Sample}

In Vancouver, the SIF is being evaluated through the Scientific Evaluation of Supervised Injection (SEOSI) cohort, which has been described previously in detail [17]. Briefly, the cohort was assembled through random recruitment of IDU from within the SIF. Since 1 January 2004, 1083 participants had been recruited to the SEOSI cohort. Our period of analysis was from 1 January 2004 to 31 January 2008.

\section{Data Collection and Measures}

SEOSI participants are asked to provide a venous blood sample for human immunodeficiency virus (HIV) and hepatitis $\mathrm{C}$ virus (HCV) testing and to complete an interviewer-administered questionnaire at baseline and at semi-annual follow-up visits. For the diagnosis of HIV, enzyme immunoassay (EIA) technology was used. First, detection of antibodies to HIV was tested by enzyme-linked immunosorbent assay (ELISA). All positive tests were confirmed by Western Blot. For the diagnosis of HCV, EIA technology was also employed, where the detection of antibodies to HCV was first assessed by the ELISA test. All positive tests were confirmed by polymerase chain reaction (PCR). The questionnaire takes approximately 45 minutes to complete and collects information on a range of topics, including socio-demographics, drug use patterns, personal history and the uptake of social services.

Since health service use may be over-reported by IDU $[2$, 12], informed consent that was obtained from all participants included a request to perform linkages with administrative health databases. In this study, a linkage to St. Paul's Hospital ED database was performed to track patient use. St. Paul's Hospital is considered to serve the majority of IDU residing in Vancouver's DTES. To be considered an ED visit for a CIRI, the following International Classification of Disease (ICD) 9 codes (Abscess and/or Cellulitis: 682.9) and the ICD 10 codes (Abscess: G061, G062, L020, L021, L022, L024, J851 and Cellulitis: L0300, L0310, L0311, L032, L0335, L038) from a patient's hospital records were used. The ICD is a World Health Organization endorsed international standard for classification of diagnoses of disease that is of use for epidemiological and clinical research and healthcare management $[18,19]$.

In addition, a linkage to the SIF database was performed to examine nurse contact and referrals issued. For this task, we conducted a record linkage to each participant's record in the SIF database to examine whether the nurse placed an entry in the SIF database indicating that they referred the participant to hospital prior to the censor or event date. We also examined whether individuals who were referred by nurses were more likely to be subsequently hospitalized within three days using in-patient hospital record linkage. We did not examine nurse-identified CIRI at the SIF in this analysis. The Research Ethics Board at the University of British Columbia approved this study.

\section{Statistical Analysis}

The endpoint for these analyses was an ED visit for a CIRI. Since females are known to display different patterns of health care utilization [10, 11], as well as CIRI development [20], we examined baseline characteristics stratified by sex and factors associated with an ED visit for a CIRI in two separate models also stratified by sex. Variables considered included: age, sex (female $v s$ male), currently residing in the DTES (yes $v s$ no), requiring assistance with injection (yes $v s$ no), living in unstable housing (yes $v s$ no), daily cocaine injection (yes $v s$ no), daily heroin injection (yes $v s$ no), daily crack injection (yes $v s$ no), daily speedball injection (yes vs no) and being HIV-positive (yes $v s$ no). Variables refer to behaviour during the last six months unless otherwise specified. Living in unstable housing refers to living in a single room occupancy (SRO) hotel, shelter, recovery or transition house or having no fixed address. We also examined whether, at the SIF, a nurse referral to hospital was associated with an increased likelihood of ED use for a CIRI. In subanalyses, we undertook an additional comparison where we investigated gender further and stratified males into men who have sex with men (MSM) and non-MSM.

Variable selection was based on previously published literature on CIRI or hospitalization among IDU in our setting $[1,2,10]$. Variables associated with an increased risk of ED visit for a CIRI were examined using unadjusted and adjusted Cox proportional hazard regression analyses. All behavioural variables were time-updated based on semiannual follow-up. Time zero was defined as the date of recruitment into the SEOSI study for all participants, and participants who had not attended the ED at St. Paul's Hospital were censored as of 31 January 2008. The multivariate model was fit using a fixed model that we adjusted for all variables that were statistically significant at the $p<0.050$ level in the univariate analyses. We ran two separate models, one for males and one for females, because the variable sex interacted with the variable HIV serostatus. All statistical analyses were performed using SAS 9.1 and all $p$-values were two-sided.

\section{RESULTS}

A total of 1083 individuals were recruited into the SEOSI cohort. Fifteen persons had missing data on HIV serostatus and were excluded from Cox proportional hazard analyses. Female participants comprised 315 (29\%) of the sample. The median age among SEOSI participants was statistically different $(p<0.001)$ between females and males, 35.1 years (interquartile range [IQR]: $28.7-41.5$ ) and 39.7 years (IQR: 33.7 - 45.3), respectively. Median duration of follow-up after recruitment into the cohort was 18.6 months and was not statistically different between the males and females $(p=$ $0.200)$. During the study period, 289 (27\%) participants used the ED for a CIRI. Of those with at least one visit, $81(28 \%)$ visited the ED for a CIRI once only during the study period, $54(19 \%)$ twice, $39(13 \%)$ three times, 32 (11\%) four times, $83(29 \%)$ five or more times for a total of 1213 identified visits. Individuals with an ED visit who were referred by a nurse were more likely to be hospitalized within three days $(p=0.011)$. 
The incidence density of first ED visit for CIRI among females was 23.8 per 100 person-years $(95 \%$ confidence interval [CI]: 19.3 - 29.0) and among males 19.2 per 100 person-years (95\% CI: $16.7-22.1)$. The difference between these densities did not reach statistical significance $(p=$ 0.090).

Baseline characteristics, stratified by sex, among IDU at a SIF are provided in Table 1. Unadjusted odds ratios indicate that females are more likely to be younger $(0.94$ [95\% CI: $0.93-0.96]$ ), require assistance with injection (2.27 [95\% CI: $1.73-2.97])$, be daily heroin injectors (1.46 [95\% CI: $1.12-1.90])$, be daily crack users $(2.29$ [95\% CI: $1.74-3.00])$, be daily speedball injectors (1.76 [95\% CI: 1.23 - 2.52]), be HIV-positive (1.42 [95\% CI: $1.01-1.99$ ]) and be referred to hospital by a nurse at the SIF (2.16 [95\%
CI: $1.31-3.54])$. In our subanalyses, females compared to MSM were not significantly more likely to be HIV-positive $(\mathrm{OR}=1.01$ [95\% CI: $0.61-1.67])$. Females compared to non-MSM were significantly more likely to be HIV-positive $(\mathrm{OR}=1.54$ [95\% CI: $1.08-2.20])$.

According to baseline information, those who used the ED for a CIRI were more likely to be female (odds ratio $[\mathrm{OR}]=1.71[95 \%$ CI: $1.18-2.47]$ ), be daily heroin injectors $(\mathrm{OR}=1.44$ [95\% CI: $1.00-2.07])$, be daily speedball injectors $(\mathrm{OR}=1.77$ [95\% CI: $1.12-2.79])$, be residing in the Downtown Eastside (OR $=1.51$ [95\% CI: $1.00-2.28]$ ), be HIV-positive ( $\mathrm{OR}=1.59$ [95\% CI: $1.03-2.47])$, require assistance with injection (OR $=2.01$ [95\% CI: $1.40-2.89]$ ) and be referred to hospital by a nurse at the SIF (OR $=4.69$ [95\% CI: 2.76 - 7.97]) (data not shown).

Table 1. Baseline Characteristics, Stratified by Sex, Among Injection Drug Users at a Supervised Injection Facility $(\mathrm{n}=1083)$

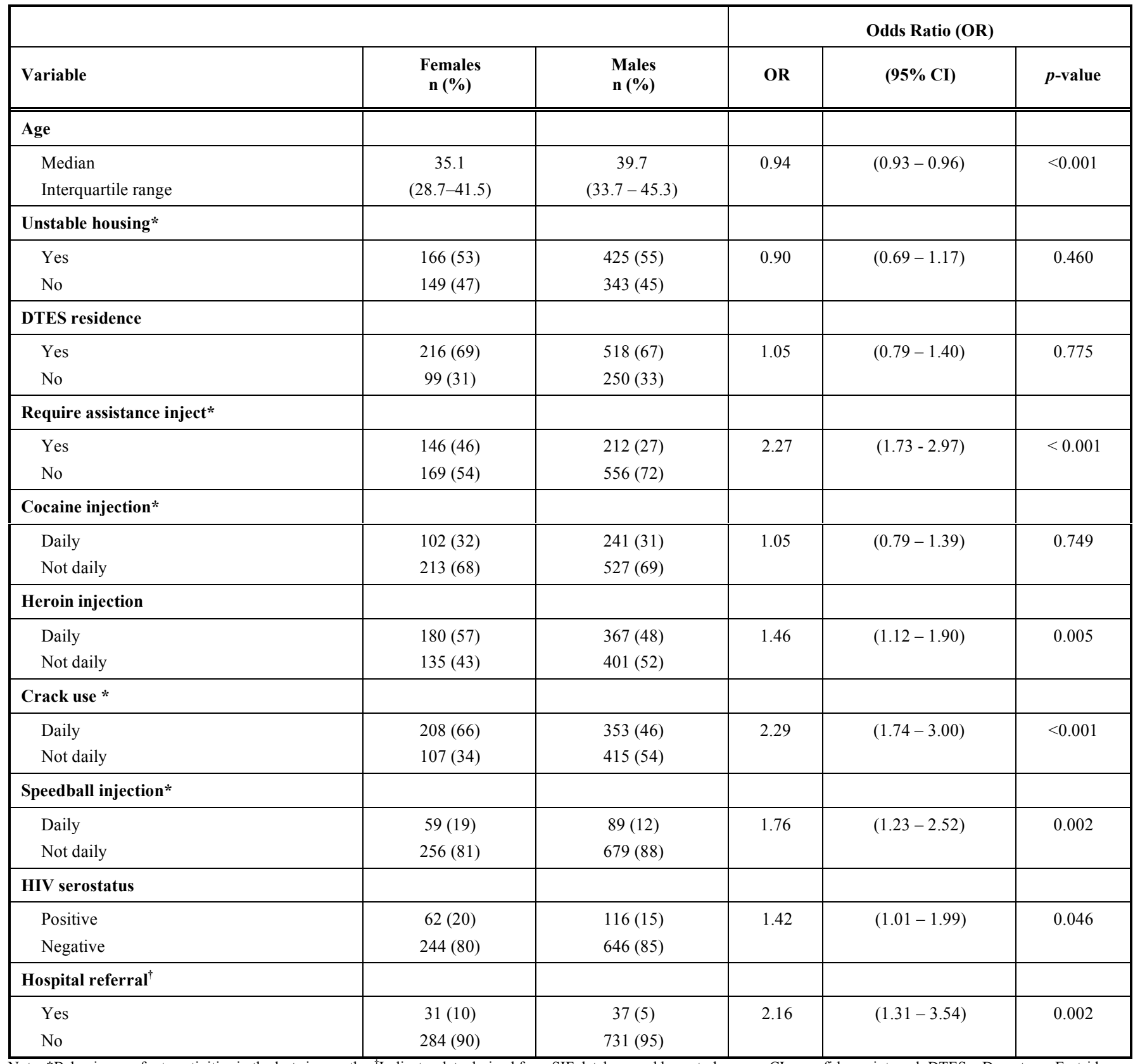


The univariate and multivariate Cox proportional hazard analyses of ED use for a CIRI among females is displayed in Table 2. In the multivariate model for females, residing in the DTES (adjusted hazard ratio $[\mathrm{AHR}]=2.06[95 \% \mathrm{CI}$ : $1.13-3.78]$ ) and being referred by a study nurse to hospital $(\mathrm{AHR}=4.48$ [95\% CI: $2.76-7.30])$ were risk factors independently and positively associated with ED use for a CIRI.

For males, the univariate and multivariate Cox proportional hazard analyses of ED use for a CIRI is shown in Table 3. The factors associated with an increased risk of ED use for a CIRI among males were requiring assistance with injection $(\mathrm{AHR}=1.38$ [95\% CI: $1.01-1.90])$, being HIV-positive (AHR $=1.85$ [95\% CI: $1.34-2.55])$ and being referred by a nurse at the SIF to hospital (AHR $=2.97[95 \%$ CI: $1.93-4.57])$.

\section{DISCUSSION}

Close to one-third of SEOSI participants visited the ED for a CIRI, yielding incidence densities of ED use for CIRI of 23.8 per 100 person-years and 19.2 per 100 person-years for females and males, respectively. The most significant predictor of ED use for a CIRI for both females and males, and in the combined Cox proportional hazard model, was being referred to hospital by a nurse from the SIF. Other determinants of ED use for a CIRI differed between female and male IDU. For females, ED use for a CIRI was also associated with residing in the DTES. For males, requiring assistance with injection and being HIV-positive were also associated with an increased risk of an ED visit for a CIRI.

Among females, residing in the DTES was a determinant of ED use for a CIRI. Females residing in the DTES can lead chaotic and unstable lifestyles that place them at an elevated risk of developing both acute and chronic health conditions [20]. A recent study by Shannon et al., found that females in the DTES frequently require emergency care, with $39 \%$ of female participants reporting an ED visit in the last six months [21]. These dynamics are complicated by the fact that outpatient or neighbourhood health clinic hours are limited and often coincide with work hours among females in this setting [1], which may result in a heavy use of the ED for health concerns. Further, this finding may reflect the close proximity of the DTES to St. Paul's Hospital $[1,2]$, but otherwise it is unclear why neighbourhood status would be associated with females and not males.

Male participants who were HIV-positive visited the ED for a CIRI significantly more often than HIV-negative participants. This is not surprising given previous reports of

Table 2. Univariate and Multivariate Cox Proportional Hazard Analyses of Time to Emergency Department Use for a Cutaneous Injection-Related Infection Among 306 Female Injection Drug Users

\begin{tabular}{|c|c|c|c|c|c|c|}
\hline Variable & \multicolumn{3}{|c|}{ Unadjusted Hazard Ratio (HR) } & \multicolumn{3}{|c|}{ Adjusted Hazard Ratio (AHR) } \\
\hline (per year older) & 1.00 & $(0.97-1.02)$ & 0.738 & & & \\
\hline \multicolumn{7}{|l|}{ Unstable housing* } \\
\hline \multicolumn{7}{|l|}{ DTES residence } \\
\hline (Yes vs No) & 2.46 & $(1.41-4.28)$ & 0.001 & 2.06 & $(1.13-3.78)$ & 0.019 \\
\hline \multicolumn{7}{|l|}{ Require assistance inject* } \\
\hline (Yes $v s$ No) & 1.56 & $(1.03-2.37)$ & 0.038 & 1.40 & $(0.92-2.13)$ & 0.122 \\
\hline (Daily $v s$ Not daily) & 1.55 & $(1.01-2.37)$ & 0.044 & 1.22 & $(0.79-1.90)$ & 0.361 \\
\hline \multicolumn{7}{|l|}{ Crack use* } \\
\hline (Daily $v s$ Not daily) & 1.42 & $(0.90-2.24)$ & 0.131 & & & \\
\hline \multicolumn{7}{|l|}{ Speedball injection* } \\
\hline (Daily $v s$ Not daily) & 1.61 & $(0.97-2.65)$ & 0.064 & & & \\
\hline \multicolumn{7}{|l|}{ HIV serostatus } \\
\hline (Positive $v s$ Negative) & 1.01 & $(0.62-1.66)$ & 0.958 & & & \\
\hline \multicolumn{7}{|l|}{ Hospital referral $^{\dagger}$} \\
\hline
\end{tabular}

Note: *Behaviours refer to activities in the last six months. Indicates data derived from SIF database and by a study nurse. Model was fit adjusted for all variables $p<0.05$ in unadjusted analyses. $\mathrm{CI}=$ confidence interval, DTES $=$ Downtown Eastside. 
Table 3. Univariate and Multivariate Cox Proportional Hazard Analyses of Time to Emergency Department Use for a Cutaneous Injection-Related Infection Among 762 Male Injection Drug Users

\begin{tabular}{|c|c|c|c|c|c|c|}
\hline \multirow[b]{2}{*}{ Variable } & \multicolumn{3}{|c|}{ Unadjusted Hazard Ratio (HR) } & \multicolumn{3}{|c|}{ Adjusted Hazard Ratio (AHR) } \\
\hline & HR & $(95 \% \mathrm{CI})$ & $p$-value & AHR & $(95 \% \mathrm{CI})$ & $p$-Value \\
\hline \multicolumn{7}{|l|}{ Age } \\
\hline (per year older) & 0.99 & $(0.97-1.01)$ & 0.287 & & & \\
\hline \multicolumn{7}{|l|}{ Unstable housing* } \\
\hline \multicolumn{7}{|l|}{ DTES residence } \\
\hline (Yes vs No) & 1.52 & $(1.10-2.11)$ & 0.012 & 1.19 & $(0.83-1.72)$ & 0.342 \\
\hline \multicolumn{7}{|l|}{ Require assistance inject* } \\
\hline (Yes $v s \mathrm{No})$ & 1.59 & $(1.16-2.17)$ & 0.004 & 1.38 & $(1.01-1.90)$ & 0.043 \\
\hline (Daily $v s$ Not daily) & 1.13 & $(0.85-1.51)$ & 0.385 & & & \\
\hline \multicolumn{7}{|l|}{ Crack use* } \\
\hline (Daily $v s$ Not daily) & 1.46 & $(1.10-1.94)$ & 0.008 & 1.30 & $(0.97-1.74)$ & 0.079 \\
\hline \multicolumn{7}{|l|}{ Speedball injection* } \\
\hline (Daily $v s$ Not daily) & 1.28 & $(0.82-2.02)$ & 0.281 & & & \\
\hline \multicolumn{7}{|l|}{ HIV serostatus } \\
\hline (Positive $v s$ Negative) & 1.90 & $(1.38-2.61)$ & $<0.001$ & 1.85 & $(1.34-2.55)$ & $<0.001$ \\
\hline \multicolumn{7}{|l|}{ Hospital referral ${ }^{\dagger}$} \\
\hline
\end{tabular}

Note: *Behaviours refer to activities in the last six months. ${ }^{\dagger}$ Indicates data derived from SIF database and by a study nurse. Model was fit adjusted for all variables $p<0.05$ in unadjusted analyses. $\mathrm{CI}=$ confidence interval, DTES $=$ Downtown Eastside.

increased hospital use among HIV-positive IDU in this setting [22, 23]. An increased susceptibility to bacterial infections [1] and other opportunistic infections among HIVpositive individuals may contribute to the greater risk of ED use for a CIRI observed among HIV-positive males during the study period [24]. It is possible that this study was underpowered to detect this association among females. In addition, these males may have continued high risk injection practices $[25,26]$, as indicated by our finding that requiring assistance with injecting was also a predictor of ED use for a CIRI among males. Requiring assistance with injecting has been reported to be related to an elevated risk of HIV [20, $25,26]$.

Importantly, the strongest predictor of ED use for a CIRI, which was also the only significant predictor for both females and males, was being referred to hospital by a nurse working at the SIF. This finding is of significance given that a goal of the SIF is to provide assessment and referral of CIRI onsite and given that the ED provides unique CIRI treatment that is not available at the SIF (e.g., incision and drainage or intravenous antibiotic therapy). Findings from this study demonstrate that nurses at the SIF play a critical role in referring individuals who use the SIF to the ED. Further, results indicate that individuals referred to the ED were more likely to be hospitalized within three days. This suggests that nurses are referring individuals to the hospital who have severe infections requiring hospitalization. Infectious complications among IDU that may require hospitalization include osteomyelitis and endocarditis [2]. However, if timely referrals are made before an infection has escalated to a point of requiring a lengthy and expensive hospitalization and if individuals can avoid hospitalization or are hospitalized for a shorter period of time, then these referrals may ultimately result in saving healthcare dollars. However, future analyses are needed to confirm the effect of SIF nurse referral on hospital costs, as well as the impact of larger SIF programs on the incidence of CIRI.

There were limitations to this analysis that need to be considered when interpreting these data. We only included ED data from one hospital in this study, which would have resulted in an underestimation of ED use as some participants would have accessed the ED in other hospitals. However, St. Paul's Hospital serves the majority of the IDU in this setting [2]. Although there were differences between males and females in our study, some trends were similar, and there may have been inadequate numbers of females to produce statistically significant differences. Also, our study relies on self-report to obtain drug use and other behavioural variables. However, self-report among IDU related to drug use and behavioural factors is considered to be generally 
valid [17], and ED use and nurse referrals were accessed directly from databases within the ED and the SIF, respectively. Further, inconsistencies in ICD reporting may have affected our results; however, ICD reporting is considered to be a valid method of disease classification [18], and there is no reason to suggest that this reporting bias would be different between groups in this study. The SEOSI cohort was randomly recruited from within the SIF and therefore is representative of IDU using Vancouver's SIF; results from this study may not be appropriate to generalize to IDU in other settings. An additional limitation is that we did not have any information on the particular role of nurses in terms of assessment of CIRI or severity of CIRI; therefore, further research is required to improve our ability to elucidate the role that nurses play in referral to emergency departments for CIRI treatment.

\section{CONCLUSION}

In summary, we found high levels of ED use for CIRI among both female and male IDU who use the SIF. Residing in the DTES and being referred to hospital by a nurse at the SIF was associated with an increased risk of an ED visit for a CIRI among females. For males, requiring assistance with injection, being HIV-positive and being referred to hospital by a nurse at the SIF was associated with an increased risk of an ED visit for a CIRI. Results from this study support the need for a better understanding of how health care workers in different settings may work together to provide more efficient, streamlined and cost-effective treatment for CIRI. Moreover, our findings suggest that the SIF could play a more intensive role in providing primary and secondary level services that could reduce both ED and in-hospital utilization for CIRI treatment.

\section{CONFLICT OF INTEREST}

All authors do not have conflicts of interest to declare.

\section{ACKNOWLEDGEMENTS}

The authors wish to thank the participants in SEOSI and the staff at Insite, the Portland Hotel Society, and Vancouver Coastal Health (Chris Buchner, David Marsh and Heather Hay.) We also thank all current and past SEOSI staff. We would also like to thank Deborah Graham, Caitlin Johnston, Steven Kain, Daniel Hawkins and Calvin Lai for their research assistance. Viviane Dias Lima and Karissa Johnston contributed statistical expertise. The evaluation of the SIF was originally made possible through a financial contribution from Health Canada, although the views expressed here do not reflect the official policies of Health Canada. This work was supported by the Canadian Institutes of Health Research (HPR-85526 and RAA-79918) and Vancouver Coastal Health. TK, MT and ELS are supported by the Michael Smith Foundation for Health Research; TK and ELS are supported by the Canadian Institutes of Health Research.

\section{REFERENCES}

[1] Kerr T, Wood E, Grafstein E, et al. High rates of primary care and emergency department use among injection drug users in Vancouver. J Public Health 2005; 27(1): 62-6.
[2] Palepu A, Tyndall MW, Leon H, et al. Hospital utilization and costs in a cohort of injection drug users. Can Med Assoc J 2001; 165(4): 415-20.

[3] Takahashi TA, Baernstein A, Binswanger IA, Bradley K, Merrill JO. Predictors of hospitalization for injection drug users seeking care for soft tissue infections. J Gen Intern Med 2007; 22(3): 382-8.

[4] City of Vancouver News Release. Mayor Releases Draft Discussion Paper on Drug Strategy, 2000 Nov 21.

[5] Irish C, Maxwell R, Dancox M, et al. Skin and soft tissue infections and vascular disease among drug users, England. Emerg Infect Dis 2007; 13(10): 1510-1.

[6] Centre for Disease Control and Prevention (CDC). Soft tissue infections among injection drug users--San Francisco, California, 1996-2000. MMWR Morb Mortal Wkly Rep 2001; 50(19): 381-4.

[7] Hope V, Kimber J, Vickerman P, Hickman M, Ncube F. Frequency, factors and costs associated with injection site infections: findings from a national multi-site survey of injecting drug users in England. BMC Infect Dis 2008; 8: 120.

[8] Wood E, Kerr T, Montaner JS, et al. Rationale for evaluating North America's first medically supervised safer-injecting facility. Lancet Infect Dis 2004; 4(5): 301-6.

[9] Wood E, Tyndall MW, Zhang R, Stoltz JA, Montaner JSG, Kerr T. Attendance at supervised injection facilities and use of detoxification services. N Engl J Med 2006; 354(23): 2512-4.

[10] Lloyd-Smith E, Kerr T, Hogg RS, Li K, Montaner JSG, Wood E. Prevalence and correlates of abscesses among a cohort of injection drug users. Harm Reduct J 2005; 2: 24.

[11] Spijkerman IJ, van Ameijden EJ, Mientjes GH, Coutinho RA, van den Hoek A. Human immunodeficiency virus infection and other risk factors for skin abscesses and endocarditis among injection drug users. J Clin Epidemiol 1996; 49(10): 1149-54.

[12] Floris-Moore M, Lo Y, Klein RS, et al. Gender and hospitalization patterns among HIV-infected drug users before and after the availability of highly active antiretroviral therapy. J Acquir Immune Defic Syndr 2003; 34(3): 331-7.

[13] Kimber J, Dolan K, van Beek I, Hedrich D, Zurhold H. Drug consumption facilities: an update since 2000. Drug Alcohol Rev 2003; 22(2): 227-33.

[14] Lorvick J, Kral AH, Seal K, Gee L, Edlin BR. Prevalence and duration of hepatitis $\mathrm{C}$ among injection drug users in San Francisco, Calif. Am J Public Health 2001; 91(1): 46-7.

[15] Dolan K, Kimber J, Fry C, Fitzgerald J, McDonald D, Frautmann F. Drug consumption facilities in Europe and the establishment of supervised injecting centres in Australia. Drug Alcohol Rev 2000; 19: $337-46$.

[16] Wright NM, Tompkins CN. Supervised injecting centres. Br Med J 2004; 328(7431): 100-2.

[17] Wood E, Kerr T, Lloyd-Smith E, et al. Methodology for evaluating Insite: Canada's first medically supervised safer injection facility for injection drug users. Harm Reduct J 2004; 1:9.

[18] Quan H, Parsons G, Ghali WA. Validity of procedure codes in International Classification of Diseases, 9th revision, clinical modification administrative data. Med Care 2004; 42(8): 801-9.

[19] International Statistical Classification of Diseases and Related Health Problems. Tenth Revision ed. Geneva, Switzerland: WHO; 1992.

[20] Lloyd-Smith E, Wood E, Zhang R, Tyndall MW, Montaner JS, Kerr T. Risk factors for developing a cutaneous injection-related infection among injection drug users: a cohort study. BMC Public Health 2008; 8: 405 .

[21] Shannon K, Bright V, Duddy J, Tyndall MW. Access and utilization of HIV treatment and services among women sex workers in Vancouver's downtown eastside. J Urban Health 2005; 82(3): 488-97.

[22] Flanigan TP, Hogan JW, Smith D, et al. Self-reported bacterial infections among women with or at risk for human immunodeficiency virus infection. Clin Infect Dis 1999; 29(3): 608-12.

[23] Selwyn PA, Alcabes P, Hartel D, et al. Clinical manifestations and predictors of disease progression in drug users with human immunodeficiency virus infection. N Engl J Med 1992; 327(24): 1697-703. 
[24] Archibald CP, Ofner M, Strathdee SA, et al. Factors associated with frequent needle exchange program attendance in injection drug users in Vancouver, Canada. J Acquir Immune Defic Syndr 1998; 17(2): 160-6.

[25] O'Connell JM, Kerr T, Li K, et al. Requiring help injecting independently predicts incident HIV infection among injection drug users. J Acquir Immune Defic Syndr 2005; 40(1): 83-8.
[26] Wood E, Spittal PM, Kerr T, et al. Requiring help injecting as a risk factor for HIV infection in the Vancouver epidemic: Implications for HIV prevention. Can J Public Health 2003; 94(5): $355-9$.

Received: June 30, 2011

(C) Lloyd-Smith et al.; Licensee Bentham Open.

This is an open access article licensed under the terms of the Creative Commons Attribution Non-Commercial License (http://creativecommons.org/licenses/ by-nc/3.0/) which permits unrestricted, non-commercial use, distribution and reproduction in any medium, provided the work is properly cited. 